\section{Signaling insulin resistance in obesity}

\section{By Lauren Martz, Staff Writer}

Researchers at Monash University have made a case for PEDF as a missing link between obesity and insulin resistance that leads to diabetes. ${ }^{1}$ However, the adipose-secreted cytokine has many biological roles, which could make it difficult for scientists to develop an inhibitor that only alters PEDF's insulin-resistance effects in obesity.

According to the Centers for Disease Control and Prevention (CDC), the prevalence of obesity in U.S. adults diagnosed with diabetes was 85.2\% in 1999-2002. ${ }^{2}$ Finding a mechanistic link between the two conditions could lead to ways to prevent diabetes in the obese population.

To address this issue, Matthew Watt, associate professor of physiology at Monash, and colleagues set out to identify highly abundant secretory proteins in the plasma of obese and insulin-resistant mice. They found that mice on a high-fat diet developed hyperglycemia and had higher plasma levels of pigment epithelium derived factor (SERPINF1; PEDF) compared with mice on a low-fat diet.

The connection to glucose metabolism was established through in vitro experiments showing that administering PEDF to skeletal muscle from lean mice reduced insulin-stimulated uptake of glucose.

In addition, caloric restriction led to weight loss and reduced PEDF levels in obese mice, whereas lean mice injected with PEDF had decreased insulin sensitivity and insulin-stimulated glucose uptake-similar to mice fed a high-fat diet.

Finally, obese mice given an anti-PEDF antibody had improvements in whole-body insulin sensitivity that were independent of changes in body mass.

The work was published in Cell Metabolism.

"If this protein turns out to be the root cause of insulin resistance in obese patients, it could offer major benefits. This is particularly attractive for prediabetic patients to prevent or slow the progression from insulin resistance to type 2 diabetes," said Brian Lavan, head of biology at Metabolex Inc.

Metabolex's MBX-102, a peroxisome proliferation-activated receptor- $\gamma$ (PPARG; PPAR $\gamma$ ) antagonist, is in Phase II testing to treat type 2 diabetes.

Leonie Heilbronn, research officer at the Garvan Institute, agreed, adding that "reducing circulating PEDF levels has the potential to improve insulin sensitivity in people with metabolic syndrome and diabetes, and potentially in all obese individuals."

\section{Translational reliance}

Still unknown is whether PEDF's function in mice is mirrored in humans. History is not on PEDF's side, as previous work on related proteins secreted from adipocytes has so far failed to translate from rodents into humans.

Lavan noted that PEDF "isn't the first molecule described in this way. And, because of what we've seen in the past, I will caution that it might not translate to humans." He told SciBX that other adipocytesecreted proteins have been shown to be related to insulin resistance in mice but their function in the human condition remains unclear.

As a case in point, Lavan cited resistin, which a team at the University of Pennsylvania School of Medicine first identified in mice in 2001 as an adipocyte-secreted protein contributing to insulin resistance. ${ }^{3}$ Although the discovery received significant attention, subsequent efforts to characterize the role of the human version of the protein in insulin resistance have been more complex than expected. ${ }^{4}$

"There is not always a relationship between what is observed in a mouse model and what is observed in humans," said Heilbronn. For PEDF, she suggested "studies in obese and lean individuals to address whether the levels of PEDF are also altered in serum and adipose tissue and then to test whether changing these levels changes insulin sensitivity."

If the PEDF results translate from mice to humans, there is still the question of how best to block the protein's activity. The issue, said Lavan, "is complicated by the fact that you are dealing with a $50 \mathrm{kDa}$ glycoprotein in circulation. One option is to use a neutralizing antibody to interact directly with PEDF, and another would be to target the protein's receptor."

Moreover, because PEDF has wide-ranging biological activities, hitting the protein raises the potential for off-target effects.

"PEDF has been shown to be involved in neuroprotection, antioxidation, antiangiogenesis and anti-inflammation, so neutralizing this protein could have potential downsides and could even promote tumor growth or formation," noted Lavan. "It is hard to predict the effects of blocking PEDF, so this will have to be thoroughly addressed preclinically before it progresses to patients."

Watt agreed. "We need to understand how PEDF signals. Specificity of action will be important given PEDF's known antiangiogenic and neuroprotective roles. There seem to be several receptors," he said.

In fact, for insulin resistance, the receptor or receptors for PEDF have "not been well characterized," said Lavan. "It is a nonclassical signaling molecule that is not well understood and could have pleiotropic effects, making it a difficult target."

Jeffrey Cleland, CEO of metabolic disease and endocrine disorder company Versartis Inc., added that there is a relationship between lack of 


\section{TARGETS \& MECHANISMS}

PEDF expression and age-related macular degeneration (AMD). "Therapeutic approaches to blocking PEDF will need to take into account the potential for accelerating AMD, the leading cause of blindness," he said.

\section{Is PEDF enough?}

Even if the effects of PEDF inhibition could be limited to the obesitydiabetes axis, some researchers expect more targets will have to be hit to have an effect on the disease.

Watt noted that other cytokines secreted by adipocytes are also expected to contribute to insulin resistance. Adipocytes are fat-storage cells that secrete various proteins to control metabolism. As the cells grow because of obesity and increased fat levels, their protein-secretion profile can change.

"The fat cell releases several factors known to cause insulin resistance," said Watt. "If those other factors are also elevated and play an important role in the human," blocking PEDF alone may not be enough.

Other cytokines involved in the relationship between obesity and insulin resistance include tumor necrosis factor- $\alpha$ (TNF- $\alpha$ ), monocyte chemoattractant protein-2 (MCP-2) and interleukin-1 $\beta$ (IL-1 $\beta$ ).

The hope is that PEDF could be causing a more significant effect on insulin resistance than the other factors or could be acting earlier during the course of disease.
Indeed, according to Lavan, this latter aspect could make PEDF a primary target. "The benefit of targeting this protein, according to the researchers, is that it could be a causative agent in insulin resistance that acts early on. If that is the case, then blocking this step could block the bad effects before they start," he said.

Watt told SciBX that the team's findings in mice have not been patented and are not available for licensing.

Martz, L. SciBX 2(30); doi:10.1038/scibx.2009.1166

Published online Aug. 6, 2009

\section{REFERENCES}

1. Crowe, S. et al. Cell Metab.; published online July 7, 2009; doi:10.1016/j.cmet.2009.06.001

Contact: Matthew Watt, Monash University, Clayton, Victoria, Australia e-mail: matthew.watt@med.monash.edu.au

2. Eberhart, M. et al. MMWR Morb. Mortal. Wkly. Rep. 53, 1066-1068 (2004)

3. Steppan, C. et al. Nature 409, 307-312 (2001)

4. Janke, J. et al. Obes. Res. 10, 1-5 (2002)

\section{COMPANIES AND INSTITUTIONS MENTIONED}

Centers for Disease Control and Prevention, Atlanta, Ga.

Garvan Institute, Darlinghurst, Sydney, Australia

Metabolex Inc., Hayward, Calif.

Monash University, Clayton, Victoria, Australia

University of Pennsylvania School of Medicine, Philadelphia, Pa. Versartis Inc., Redwood City, Calif. 